\title{
Deep Venous Thrombosis in a Patient with
} a Moderate Pretest Probability and a Negative D-Dimer Test: A Review of the Diagnostic Algorithms

This article was published in the following Dove Press journal:

Journal of Blood Medicine

\author{
Rasha A Al-Khafaji (D) ${ }^{1,2}$ \\ Louise Schierbeck' \\ 'Cardiology Department, Nordsjælland \\ (North Zealand) University Hospital, \\ Hillerød, Denmark; ${ }^{2}$ Endocrinology and \\ Nephrology Department, Nordsjælland \\ (North Zealand) University Hospital, \\ Hillerød, Denmark
}

\begin{abstract}
Modern diagnostic strategies of venous thromboembolism (VTE) have been developed. In this review, the diagnostic algorithms for deep-vein thrombosis (DVT) and their parameters are discussed individually in the context of reporting a case of DVT in a 43-yearold Caucasian female with a moderate pretest probability stratified by Wells' score and a negative high quality D-dimer test. The patient was on treatment with Xarelto (rivaroxaban), $20 \mathrm{mg}$ PO daily at the time of presentation. The diagnosis was verified through a complete lower limb ultrasound (US). This case highlights the diagnostic challenges and pitfalls of the current algorithms, especially those seen in a subgroup of patients such as patients with cancer, pregnancy, recurrent VTE or are on anticoagulation therapy at the time of presentation. The diagnosis of DVT is less plausible in a patient who is on anticoagulation therapy, but physicians should be aware of such a possibility. Physicians should also know in advance the numerous clinically relevant limitations of D-dimer testing before interpreting the results. Unifying the current diagnostic strategies, modifying the current Wells' score and using the protocol of a whole-leg compression US instead of the limited US protocol are among the several cautious suggestions that have been proposed based on this review to possibly decrease the incidence of missed DVT.
\end{abstract}

Keywords: compression ultrasound, recurrent thrombosis, rivaroxaban, Wells' score

\section{Introduction}

VTE is the third leading vascular diagnosis after myocardial infarction and stroke. $^{1,2}$ It is a complex multifactorial disease and an important cause of preventable mortality and morbidity. ${ }^{3}$ The economic burden of VTE spans from the loss of economic output due to premature mortality to the medical costs in treating the acute event and the costs of complications and long-term morbidity. $^{3}$

VTE includes two major clinical manifestations; DVT and pulmonary embolism (PE). DVT is the most common clinical manifestation, where a blood clot typically forms in the deep veins of the leg and/or pelvis. Recurrent thrombosis and post-thrombotic syndrome (PTS) are known complications of DVT. PE is the most serious manifestation and it occurs when a blood clot becomes lodged in the arteries of the lung. ${ }^{1,4-10}$ PE can cause death and chronic thromboembolic pulmonary hypertension (CTEPH). ${ }^{1-10}$
Correspondence: Rasha A Al-Khafaji; Louise Schierbeck

Tel +45 3। 32 I। 89; +45 48 2952 I5

Email rasha.alkhafaji2@gmail.com;

louise.schierbeck@gmail.com 


\section{Overview of the Current Diagnostic Strategy for VTE}

VTE can be predisposed by different risk factors whose effects vary according to age, gender and the presence of other potentiating factors at the time of clot formation. Recent surgery, active cancer, trauma, leg paresis and nursing home confinement are considered major risk factors. Pregnancy, oral contraceptives and oral postmenopausal hormone therapy are well-known factors for thrombosis in females. $^{5,6}$

The major risk factors have been incorporated alongside the tremendous body of evidence that has been generated in the previous decades to develop guidelines and algorithms, which can be used in prevention, diagnosis and treatment of VTE. ${ }^{5,6,8,11-13}$ The modern diagnostic strategies involve sequential testing as a D-dimer and/or imaging depending on the VTE risk stratification by Wells score. ${ }^{2,8,14}$

\section{The Case Report}

A 43 year old Caucasian female sought repeated medical attention from her general practitioner (GP) on the third day of her symptoms with complaints of right leg swelling. She had previously sought acute medical attention by visiting the accident and emergency department (A\&E) on the first day of her symptoms because of noticing that the right leg was larger. But this physical finding could not be detected by the physician on-call. The patient was discharged after DVT was excluded based on a normal physical examination and a negative D-dimer test.

The patient was seen by the main author for first time in the same A\&E when she was referred by the GP for a suspected DVT. In this second admission, the patient presented with a unilateral slight visible swelling in the lower right extremity. The patient did not have fever, pain, restriction in movement, itching, syncope, chest pain, acute shortness of breath, orthopnea nor abdominal symptoms. There was no history of trauma, insect's bites, travelling, weight loss or anything out of usual in her routine activities.

The patient confirmed her clinical history; a previous verified bilateral PE by SPECT-CT 5 months earlier, a 20-year use of oral contraception, which was stopped due to PE, a maternal grandmother with PE at the age of 42. Six weeks prior to this second admission, the patient had been seen at another hospital, where (CTEPH) had been ruled out. But, the patient was nevertheless waiting for an appointment for Right Heart Catheterization (RHC) and possibly an exercise-RHC too. The patient was also waiting for the results of her thrombophilia testing and was on a follow-up schedule to exclude antiphospholipid syndrome (APS). The only current medication was rivaroxaban (Xarelto); $20 \mathrm{mg}$ daily in the last 4 months.

The physical examination revealed a $\mathrm{BMI}>37$, right lower extremity presented with slight redness, slight visible swelling up to her mid leg, grade 2 pitting edema, with $2.5 \mathrm{~cm}$ difference in circumference between the legs measured from the medial malleolus (not from the tibial tuberosity), no distended veins, no warmth, no tenderness, no muscle spasm in her lower limb, and she had bilateral normal dorsalis pedis pulses. The patient had a normal temperature, pulse, blood pressure and a respiratory rate. No elevated jugular venous pressure, no hepatojugular reflex. Auscultation of both the lungs and the heart was unremarkable.

Biochemically, there was a slight leucocytosis up to $12 \mathrm{E}^{9} / \mathrm{L}$ with a normal CRP and a normal D-dimer test. The D-dimer is routinely measured by the nurse at our center when a patient is referred suspected of DVT before these patients are seen by a doctor. D-dimer was measured by the STA-Liatest D-Di PLUS kit, reference 00662, which is an immune-turbidimetric assay for the quantitative determination of D-dimer in venous plasma. ECG was normal.

Because of the somewhat vague symptoms and Xarelto, the assessment of having DVT was less plausible. The pitting edema received 1 point and despite there was no full leg swelling, but 1 point was also given for this sign according to the Wells' score in the electronic system in Denmark (Table 1). Because of a total score 2, there was a moderate risk of having DVT according to the 3 risk stratification module. The main author decided to refer the patient for a complete right lower limb Doppler US the next morning. The US confirmed thrombosis in the popliteal and calf veins of the affected right limb. Treatment with Xarelto was stopped and low molecular heparin (Innohep) was initiated.

On a consented follow-up of the patient's electronic record for this publication at the time of writing the original manuscript: Thrombophilia tests and APS tests were both negative, and the RHC later confirmed the development of CTEPH, which the findings barely fulfilled the diagnostic criteria. The patient was not under investigation for cancer because of lack of clinical suspicion and the patient was in relevant treatment.

\section{Discussion}

The clinical diagnosis of symptomatic VTE is difficult because the clinical presentations overlap with other 
Table I Wells' Score for Deep Venous Thrombosis

\begin{tabular}{|l|l|l|l|}
\hline $\begin{array}{l}\text { DVT-Clinical Scoring system (Wells' Score Used in Denmark } \\
\text { Translated from the Danish Language }\end{array}$ & $\begin{array}{l}\text { Wells' Criteria for DVT in Other Countries Such } \\
\text { as the USA and the UK }\end{array}$ \\
\hline Active cancer, that is to say active/palliation treatment in the last 6 months & $+I$ & Active cancer Treatment or palliation within 6 months & $+I$ \\
\hline Paralysis, paresis of the lower limb, possibly a recent cast of the lower limb & $+I$ & $\begin{array}{l}\text { Bedridden recently }>3 \text { days or major surgery within I2 } \\
\text { weeks }\end{array}$ & $+I$ \\
\hline $\begin{array}{l}\text { Recent immobility }>3 \text { days, possibly a major surgical intervention in the last } \\
\text { month }\end{array}$ & $+I$ & $\begin{array}{l}\text { Calf swelling }>3 \text { cm compared to the other leg. } \\
\text { Measured I0 cm below tibial tuberosity }\end{array}$ & $+I$ \\
\hline Local tenderness along the deep veins & $+I$ & Collateral (non varicose) superficial veins present & $+I$ \\
\hline Swelling in an entire lower limb (Circumference should be measured) & $+I$ & Entire leg swollen \\
\hline $\begin{array}{l}\text { Swelling in the leg more than } 3 \text { cm compared with the asymptomatic side } \\
\text { (Measured I0 cm from the tibial tuberosity) }\end{array}$ & $+I$ & Localized tenderness along the deep venous system & $+I$ \\
\hline Pitting edema, if it is most prominent in the affected lower limb & $+I$ & Pitting edema, confined to symptomatic leg \\
\hline Dilation of the superficial veins of the affected lower limb (Not varicose veins) & $+I$ & $\begin{array}{l}\text { Paralysis, paresis, or recent plaster immobilization of } \\
\text { the lower extremity }\end{array}$ & $\begin{array}{l}+I \\
+I\end{array}$ \\
\hline Alternative diagnosis is more likely than DVT & -2 & Previously documented DVT \\
\hline
\end{tabular}

Notes: 'anish version of Wells' score, data from Lægehåndbogen. ${ }^{15}$ bWells' criteria for DVT, data from MDCalc. ${ }^{16}$ The risk factor highlighted in bold is the missing risk factor from the Wells' score, which was used in Denmark at the time of the patient's presentation to the A\&E with a suspected DVT.

diseases. Referring all patients with suspicious symptoms for definitive diagnostic imaging to rule in or out VTE would be impractical, inefficient usage of resources, time consuming and, in some cases, may expose patients to unnecessary radiation.

Therefore, there is a need for algorithms and diagnostic tests that can quickly triage the patients in daily clinical practice and thus assist physicians. ${ }^{5,8,9}$ Based on Wells' score, relevant diagnostic tests can be used. ${ }^{2,8,11-13}$ These include D-dimer measurements, leg vein compression US (CUS) used mainly for DVT, chest computed tomography pulmonary angiography CTPA, or ventilation perfusion (V/Q) lung scans for PE. . $^{8,911,12}$

The safety and cost-effectiveness of these strategies have been extensively validated. ${ }^{5,12}$ But, there are still limitations and challenges in a subgroup of patients who are elderly, pregnant, on anticoagulation therapy or have cancer and/or recurrent VTE.

\section{Pretest Probability}

Wells' clinical prediction module for DVT (Table 1) and PE enables physicians to stratify patients into high-, moderate-, or low-risk categories. ${ }^{2,11,14,17,18}$ Geneva score can also be used for PE. ${ }^{11,17,18}$ The revised Geneva score is entirely based on clinical variables and is independent of physician judgment. ${ }^{17}$
In the 3 risk stratification module of Pretest probability (PTP); a score of 0 or less are considered low risk, a score of 1-2 indicates moderate risk, and 3 or greater are high risk. The probability of DVT increases with a higher stratification score; Low (5\%), moderate (17\%) and high $(53 \%) .{ }^{12}$ For simplification purposes the Wells' score can also stratify the patient's risk into 2 groups; DVT unlikely (Wells' score $<2$ ) or DVT likely (Wells' score $\geq 2$ ). ${ }^{14,19}$ In this type of stratification, the risk of having DVT is $6 \%$ in the unlikely group while the likely group has a risk of $28 \%{ }^{12}$ In Denmark, a 3 risk stratification module is used.

In Wells' score, the presence of each clinical feature is given a positive score of +1 . A score of -2 is given if an alternative diagnosis is highly likely, which mainly depends on the individual physician's judgment (Table 1). In an observational study ${ }^{20}$ involving 117 emergency departments (116 in France and 1 in Belgium) to assess the implementation of the international diagnostic strategies of PE by physicians in routine practice, it was shown that when physicians ruled out PE, they used appropriate diagnostic criteria in only $43 \%$ of the observed 1529 patients. ${ }^{20}$ This was attributed to variable factors, which could alter the clinical judgment; older age, other underlying pathology as heart failure, lung disease, current or recent pregnancy and currently receiving anticoagulant treatment. ${ }^{17,20}$ 
There is a difference between the used Wells' score in the UK, the USA and Denmark at the time when the patient was admitted to the A\&E. The Wells' score used in Denmark lacks a point for a previously documented DVT, which is highlighted in bold (Table 1). A single point can alter the scoring and therefore alters the clinical decision regardless if it is according to the Danish, ASH or NICE guidelines (Figures 1,2 and 3). In hindsight, although the patient had a documented PE and not a DVT, one could argue for it to be used as a documented case of previous thrombosis and add +1 to the score. Thus, the patient would have scored 3 (High risk group) and subsequently be managed with a different algorithm (Figures 1 and 2). In patients with a high PTP, not even using a high sensitive D-dimer assay should be used for ruling-out VTE. ${ }^{21}$ Because none of the current assays have a sufficiently high negative predictive value to be used for rule-out in the "““ High"” PTP. ${ }^{14}$

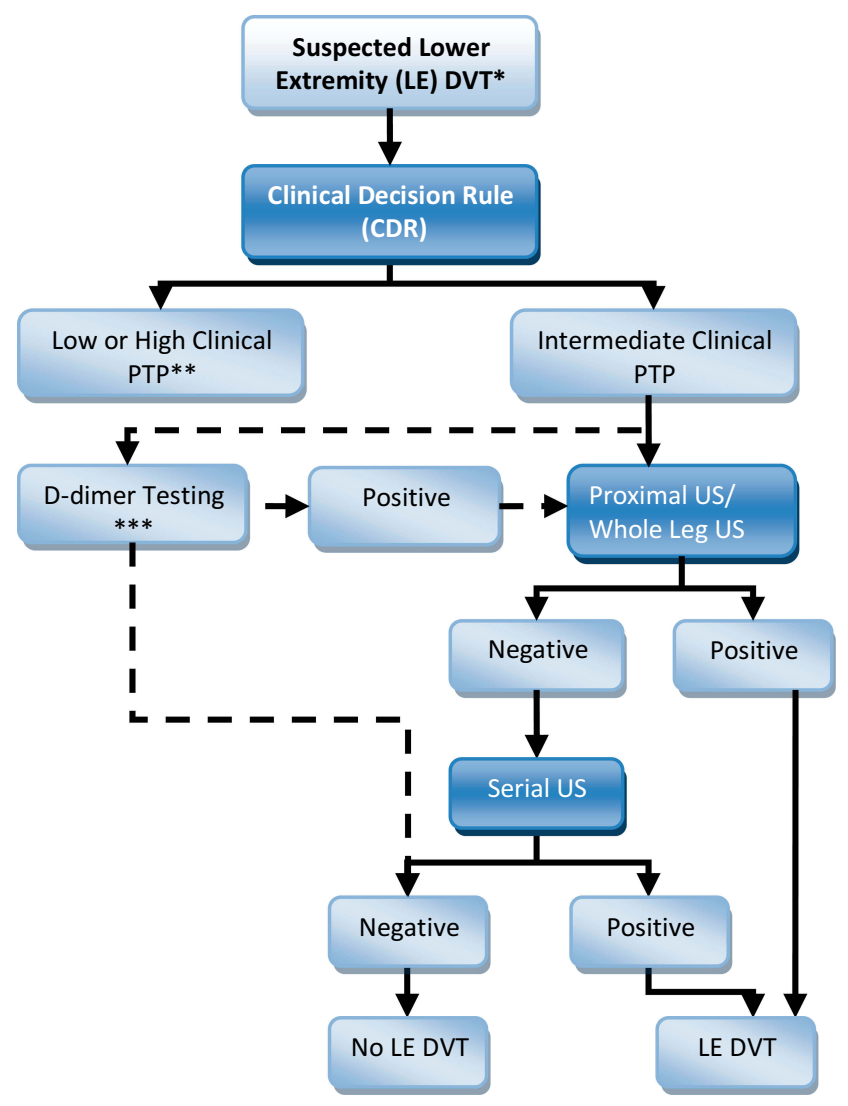

Figure I Flowchart for ASH recommendations (diagnosis of DVT for patients with intermediate PTP/prevalence [ 25\%]).

Notes: *Hemodynamically stable, nonpregnant patient. **See other algorithms. ***Highly sensitive D-dimer. Adapted with permission of American Society of Hematology, from American Society of Hematology 2018 guidelines for management of venous thromboembolism: diagnosis of venous thromboembolism, Lim W, Le Gal G, Bates S et al, Blood Adv, 2(22), 2018; permission conveyed through Copyright Clearance Center, Inc. ${ }^{13}$

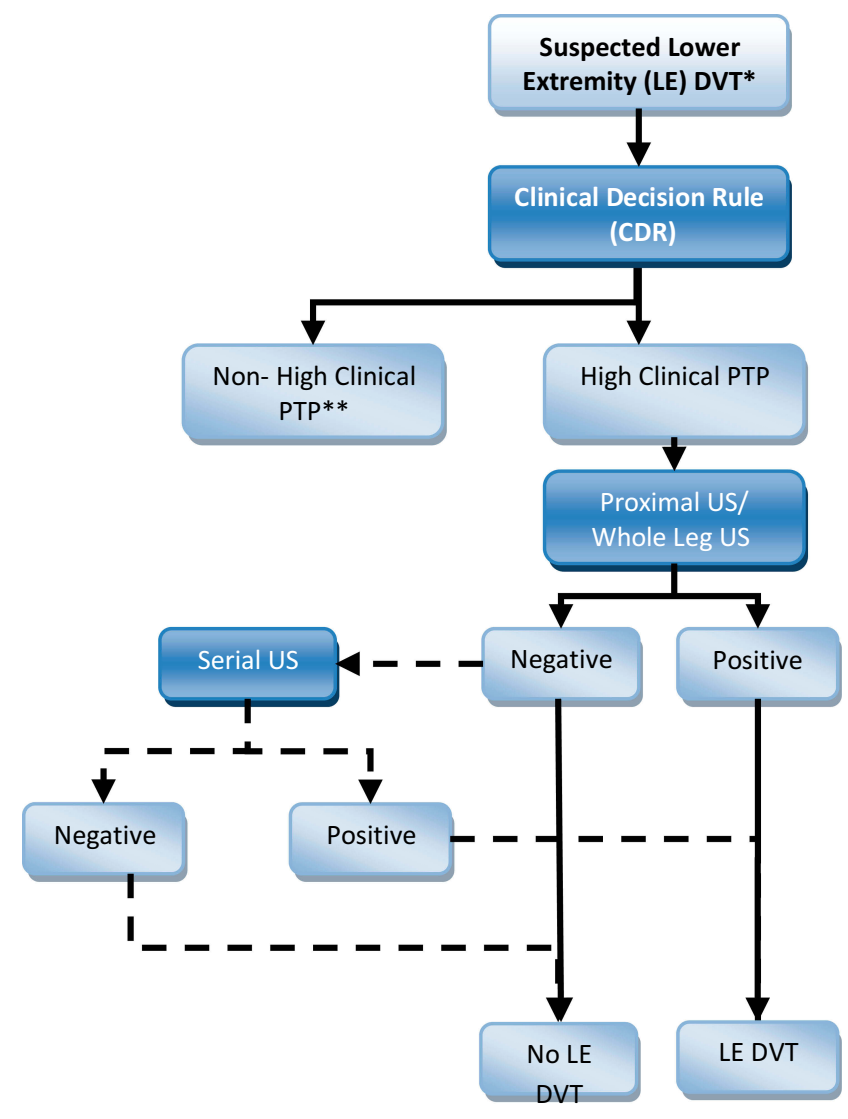

Figure 2 Flowchart for ASH recommendations (diagnosis of DVT for patients with high PTP/prevalence $[\geq 50 \%]$ ).

Notes: *Hemodynamically stable, nonpregnant patient. **See other algorithms. Adapted with permission of American Society of Hematology, from American Society of Hematology 2018 guidelines for management of venous thromboembolism: diagnosis of venous thromboembolism, Lim W, Le Gal G, Bates S et al, Blood $A d v, 2$ (22), 2018; permission conveyed through Copyright Clearance Center, Inc. ${ }^{13}$

Identifying and diagnosing atypical presentations of VTE, including isolated distal DVT (IDDVT) and isolated PE (I-PE) without DVT has become possible due to the use of diagnostic tests combined with advances in imaging technology. ${ }^{22}$ A false negative CUS of lower limb especially in asymptomatic patients is considered the major explanation of diagnosing PE without limb DVT because the thrombus has either resolved or migrated.

By analyzing 521,000 electronic records of combat patients, because they have a higher risk of developing a VTE compared to the civilian patients, it was shown that DVT and PE have overlapping risk factors. But DVT was more in patients who have the clinical picture of immobility, while PE was more in those who had chest injuries. ${ }^{23}$ This raised new questions about the natural course and pathophysiology of PE without DVT. ${ }^{23}$

Risk factors, clinical presentation and 3-month survival in each form of VTE in 5889 patients were analyzed in the Optimev study. ${ }^{22}$ It was observed that younger patients, 


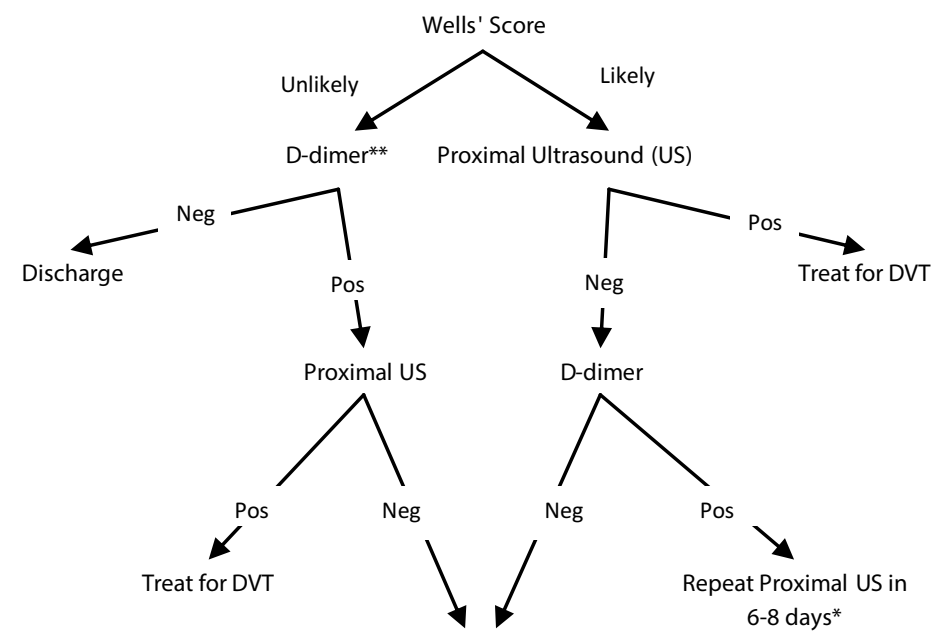

Discharge

Figure 3 Diagnostic Algorithm of DVT in outpatients in Oxford's University hospitals/UK.

Notes: Wells' score $\leq \mathrm{I}$; unlikely, score $\geq 2$; likely. *'Likely" patients who do not have a negative result in D-dimer testing, need a repeat scan of the proximal veins one week later. They remain off anticoagulation whilst awaiting this. An alternative strategy for these patients would be to extend the initial scan to the whole leg (ie to also scan the calf veins). **Patients who have already had an anticoagulant, D-dimers cannot be used as part of the diagnostic algorithm. Patients without a D-dimer result will follow the same path as if D-dimer were positive. Adapted from Oxford University Hospitals, NHS Foundation Trust. Oxford Haemophilia And Thrombosis Center Out-Patient DVT Service Protocol. Oxford: Oxford University Hospitals; 2019. Version 9.6 [updated by Curry N]. Available from: https://www.ouh.nhs.uk/services/referrals/specialistmedicine/documents/dvt-protocols.pdf. Accessed April 30, 2020. ${ }^{19}$

who underwent exploration procedures without bed confinement were likely to have PE without DVT even if they share permanent risk factors like cancer with the PE with DVT patient group. The same applies for inpatients but without being bedridden. ${ }^{22}$

PE could be a presentation of a primary hypercoagulable event rather than an embolized thrombus. ${ }^{22}$ Based on the study done by Palereti $\mathrm{G}$ et al, ${ }^{24}$ which involved 3573 patients included in the START2-Register for a VTE event; $2880(80.6 \%)$ had DVT/PE, the remaining had I-PE (19.4\%). It was found that hormonal contraception, were more prevalent in I-PE than in DVT/PE Groups. Also, older age, female gender, cancer and heart failure were risk factors for I-PE. I-PE from right-sided intra-cardiac thrombosis or simply in-situ thrombus formation are other proposing sources. ${ }^{24}$

Taking into account the previous and the clinical case, modifying the current Wells' score (Table 1) by changing the risk factor from "Previously documented DVT" to "Previously documented DVT and/or PE" could possibly result in a better performance.

\section{The Guidelines and Algorithms}

Despite multiple algorithms exist to aid physicians in daily practice, but this multiplicity may lead to incorrect application and potentially medical errors. The guidelines are not unified which adds, in certain circumstances, more challenges in diagnosing DVT. For example, a difference that exists between the NICE guidelines, which currently depends on a 2 risk stratification module (Figure 3 ) and the guidelines of the $\mathrm{ASH}$, which depends on the 3 risk stratification module (Figures 1 and 2). The former does not emphasize the diagnosis of IDDVT because proximal US is the initial preferred test, and with a negative d-dimer test a patient could be discharged (Figure 3).

It was shown in a study ${ }^{25}$ that the sensitivity of Wells' score for IDDVT was $47 \%$ and the negative and positive predictive values were $91 \%$ and $20 \%$ respectively. Also, $14.4 \%$ (13/90) patients with IDDVT had negative results on a D-dimer test. Among the patients with IDDVT and negative D-dimer, seven patients scored PTP 2 (moderate), five patients scored PTP 1 (Moderate), and only one scored PTP $<1$ (Low). Thus, in patients with moderate risk, D-dimer had a lower negative predictive value. ${ }^{25}$

In reviewing the literature, IDDVT is currently one of the most debated issues in the field of VTE and it is still a controversial grey area. ${ }^{26-29}$ There is a high degree of uncertainty about the clinical relevance of IDDVT and subsequently there is a disagreement regarding the need for diagnosing and treating. ${ }^{27,30}$ Clinical opinion is divided between that the benefits of treatment are counterbalanced by potential harm. ${ }^{29}$ 
IDDVT represents a frequent relevant medical condition; up to $50 \%$ of all lower-limb DVTs are distal. ${ }^{27,28}$ Acute IDDVT contributed $49.6 \%$ of all DVTs cases in a study cohort from an A\&E in the UK. ${ }^{29}$ DVT and IDDVT had similar risk profiles with IDDVT being more common in patients undergoing surgery. ${ }^{22}$ But IDDVT has a lower incidence of complications when compared with proximal DVT, which could be due to the rapid resolution of the thrombus in IDDVT. ${ }^{31}$

There is a reported (1\%-5.7\%) risk of proximal extension of IDDVT in studies that adopted serial proximal CUS,${ }^{27}$ while others report a $9-21.4 \% .{ }^{32}$ Infrapopliteal DVT is frequently diagnosed in subjects with $\mathrm{PE} ;{ }^{27} \mathrm{PE}$ risk was $1.6 \%$ in the CALTHRO study. ${ }^{27}$ In Sevestre's $\mathrm{M}$ et al study, ${ }^{22} 35 \%$ of the PE cases with DVT had IDDVT. $^{22}$ IDDVT may also lead to PTS disease. ${ }^{27}$ Thus, treating IDDVT is warranted. ${ }^{27}$

Currently, there are no tools available to discriminate between low- and high-risk IDDVT patients; inpatients, patients with active cancer, or patients with previous VTE. ${ }^{26}$ Anticoagulation therapy may maximally benefit those patients with extensive and/or symptomatic disease or those with higher risk for complication (unprovoked distal DVT, cancer-associated or pregnancy). ${ }^{28,29}$

Despite a significant proportion of the hospitals in the UK use only proximal CUS in suspected cases (Figure 3), ${ }^{19}$ the current UK guidance continues to recommend therapeutic anticoagulation for IDDVT. ${ }^{29}$ Regardless of whether IDDVT is to be treated with anticoagulation or not, diagnosing IDDVT is warranted. ${ }^{32}$ Taking into account the previous discussion, using a 2 stratification Wells' score module and following NICE guidelines in the management of DVT, missing the diagnosing of IDDVT is possible.

\section{D-Dimer}

The breakdown of fibrinogen and fibrin during fibrinolysis produces D-dimers. The D-dimer tests are widely used as an initial screening test in the A\&E alongside the PTP to rule out VTE or warrant further imaging (Figures 1 and 3). It is a proven cost-effective way to triage the patient. D-dimer test is highly sensitive (>95\%) in acute DVT or PE, usually with a cut-off value of $500 \mu \mathrm{g} \mathrm{FEU/1,} \mathrm{which} \mathrm{reasonably} \mathrm{rules}$ out acute VTE, particularly in patients with low or moderate PTP. ${ }^{17,33}$ By randomly assigning 552 patients to evaluate the use of D-dimer testing in the diagnosis of DVT, only 17 out of 218 negative D-dimer tested patients, returned during the follow-up. 4 with suspected PE and 13 with suspected DVT. 2 had proximal DVT on days 4 and 14 of the follow up. ${ }^{34}$

\section{A General Review in the Clinically Relevant Limitations of the D-Dimer Testing}

Serum D-dimer elevation is not specific to thromboembolic disease, and baseline D-dimer elevation can be observed in many other conditions including physiological causes such as pregnancy, puerperium, African American heritage, cigarette smoking and increasing age above $65.4,9,17$

Currently, there are more than 30 different assays being used in clinical practice, which mostly use monoclonal antibodies that target specific epitopes on cross-linked D-dimer fragments. ${ }^{21}$ Examples; Latex agglutination, enzyme-linked immunosorbent assay (ELISA) as well as a number of rapid, point-of-care D-dimer assays. ${ }^{4}$

Sensitivity and specificity of the D-dimer assays vary depending on the methodology that is being utilized for detecting D-dimer. Dagkiran $\mathrm{H}$ et $\mathrm{al}^{35}$ reported a case of severe PE with a negative D-dimer test measured by latex method. Despite the PTP was not reported in their publication, ${ }^{35}$ but by scoring it from the given published data it was a "Low" risk. According to the algorithms a Low PTP with a negative D-dimer test could rule out PE. Therefore, because of the irregularities in the quantitative determination of D-dimer, a negative D-dimer testing alone is not sufficient for excluding PE. ${ }^{35}$

Efforts in standardizing D-dimer results have not yet been successful because of the non-uniform analyte and the non-homogenous molecular structure of the D-dimer. Results may be reported using 2 different types of units: fibrinogen equivalent unit as in the test used in the reported clinical case in this review, and D-dimer unit. Also, either type of unit used for D-dimer quantification may be reported with as many as 9 different units of magnitude, including $\mathrm{mg} / \mathrm{L}, \mathrm{mg} / \mathrm{dL}, u g / \mathrm{mL}, u g / \mathrm{dL}$, etc. ${ }^{4}$ Also, different cut-off values exist among different manufacturers. Inter laboratory variation further compromises the value of the D-dimer assay. ${ }^{4}$ All these complicate the standardization further. ${ }^{17}$

The possible presence of interfering substances; Hemoglobin, triglycerides, bilirubin, rheumatoid factors and some monoclonal antibodies or simply the presence of other diseases as renal failure can all affect the D-dimer assay. Many drugs can also interfere with D-dimer assays but usually not at therapeutic levels. ${ }^{4}$ Individual patient heterogeneity in D-dimer, the clot's size and site, the timing of specimen collection in relation to the thrombotic event as well as anticoagulation and fibrinolytic therapy all affect testing results. ${ }^{4,9,17,18}$ 
There is a decreased sensitivity of the D-dimer testing in low thrombotic burden and distal DVT. ${ }^{9}$ The sensitivity of D-dimer testing for individuals with low to moderate PTP was lower for those $<60$ years old versus $>60$ with a marked difference in the same study. ${ }^{9}$ Despite the levels of D-dimer is significantly higher in patients with IDDVT than those without a thrombus, but it is significantly lower than those in patients with proximal thrombi. ${ }^{36}$ Interestingly, Singer A. et $\mathrm{al}^{36}$ also found that several IDDVTs could be missed in patients with moderate risk PTP in whom D-dimer testing was negative. ${ }^{36}$ This is because according to the algorithms, imaging is only recommended for moderate risk patients, who test positive in a highly sensitive D-dimer assay. (Figures 1,2 and 3). ${ }^{36}$

\section{D-Dimer Testing in Specific Subgroups}

Diagnostic challenges of VTE are seen in a subgroup of patients who are elderly, pregnant, on anticoagulation therapy or have cancer and/or recurrent VTE. Here, only D-dimer testing in patients on anticoagulation therapy and oncology patients with VTE are reviewed.

\section{D-Dimer Testing in Patients on Anticoagulation Therapy}

There are currently limited data for the utility of D-dimer for patients receiving anticoagulant therapy and who present with suspected recurrent PE. ${ }^{13}$ D-dimer has also been suggested to determine the length of anticoagulation therapy. This is discussed in the section reviewing imaging techniques. D-dimer levels are suppressed by all anticoagulant drugs. ${ }^{37}$ After initiating anticoagulation with heparin, the D-dimer decreases by an average of $25 \%$ upon the administration of heparin 24 hours in the treatment of acute VTE. This is enough to decrease the sensitivity of D-dimer testing from $95.5 \%$ to $89.4 \% .{ }^{17,38}$

Fondaparinux, a direct $\mathrm{Xa}$ injectable inhibitor, is used as a prophylaxis for postoperative DVT. A study ${ }^{39}$ conducted to assess the influence of Fondaparinux on D-dimer testing for DVT screening in patients undergoing total hip arthroplasty (THA) and total knee arthroplasty (TKA), revealed that only results of $\mathrm{D}$-dimer testing on postoperative day 7 was useful for DVT screening, which yielded a sensitivity of $86 \%$, but a poor specificity of $25 \%{ }^{39}$ In patients who received fondaparinux for 14 days after a THA, the incidence of DVT was $13.6 \%$ on postoperative day (POD) 1 , $27.1 \%$ on POD 4 . In patients after a TKA, the incidence of DVT was $50.0 \%$ on POD $1,58.3 \%$ on POD 4 . Therefore, the group ${ }^{39}$ concluded that the accuracy of D-dimer test was decreased by the administration of fondaparinux and it might be necessary to find a new strategy for diagnosing DVT for patients receiving fondaparinux. ${ }^{39}$

Vitamin $\mathrm{K}$ antagonists (VKAs) and direct oral anticoagulants (DOACs), which both work by different mechanisms, are globally accepted as oral anticoagulants (OAC) to prevent thrombosis and suppress D-dimer production. ${ }^{40}$ The effect of OAC on D-dimer testing depends on the dose, type, indication, context etc. Therefore, different studies give different accounts in respect to D-dimer.

Although dabigatran and rivaroxaban do not affect the D-dimer assay but the D-dimer level is likely to be low. ${ }^{37}$ When comparing Warfarin and DOAC in patients treated for VTE, the rate of positive D-dimer assessed during anticoagulation was significantly higher in patients treated with DOACs than Warfarin for VTE. ${ }^{40}$ A similar result was found in patients receiving edoxaban, a direct $\mathrm{Xa}$ oral inhibitor, as a thromboprophylactic in the postoperative period of a THA. ${ }^{41}$ The use of edoxaban was correlated with a high D-dimer level on POD 21, but not on PODs 7 and 14. This could be due to hemostasis being delayed by anticoagulants, which may result in a higher amount of local hemorrhage and thus fibrin degradation is prolonged. ${ }^{41}$

Rivaroxaban is also a direct Xa oral inhibitor. To identify the sensitivity of D-dimer in predicting DVT in the first 4 days of the postoperative period in patients receiving a TKA while on rivaroxaban $10 \mathrm{mg}$ PO as prophylaxis for the first 14 postoperative days, 200 enrolled patients received regardless of the D-dimer testing also bilateral ascending venography of the legs, which was carried out on the next day after the last dose of rivaroxaban, or earlier if symptomatic. 29 of the 200 (14.5\%) patients developed DVT, which was diagnosed through venography. ${ }^{42}$ Because all the DVTs occurred in the leg veins, decreased thrombus volume and size might result in poor accuracy of plasma D-dimer test in prediction or diagnosis of postoperative DVT. $^{42}$

A case of PE in a fully anticoagulated rivaroxaban patient with a moderate PTP for PE and a negative d-dimer test has also been reported. ${ }^{43}$ Rivaroxaban was also used by the patient in this review at the time of recurrent VTE. Whether rivaroxaban can influence the sensitivity of D-dimer testing or not, is beyond the scope of this review and requires further studies. In summary, physicians should be aware that anticoagulation therapy could influence the result of D-dimer testing and a full anticoagulation with rivaroxaban does not exclude the risk of recurrent VTE. 


\section{D-Dimer Testing in Cancer Patients with VTE}

A mini-review of the literature linking D-dimer, with VTE and cancer is warranted because VTE in cancer patient proposes diagnostic and prediction challenges. Also, it is a prognostic parameter for a shorter survival and represents the leading causes of death in cancer patients. The intrinsic thrombogenicity of cancer and/or the immobility caused by general weakness, chemotherapy, and surgery contribute to VTE formation. ${ }^{44}$ Cancer's site, grade, and histological type are identifiable risk factors for VTE. ${ }^{45}$

There is a $10-15 \%$ risk or more in developing VTE in the first 6 months in ambulatory patients with solid cancers. Thus they may benefit from thromboprophylaxis. ${ }^{45}$ Khorana score, Vienna modification of the Khorana score, PROTECHT score and CONKO score are different scores, which are being used in practice to detect VTE in cancer patients undergoing chemotherapy as outpatients. However, because of the inadequate predictive power their performance is limited.

Because only tumour-site category in the Khaorana was significantly associated with the risk of developing VTE in the first 6 months in the CATS and MICA studies and D-dimer testing has been introduced into routine practice as a VTE risk predictor (Figures 1 and 3), a novel simple clinical prediction model for predicting VTE in ambulatory patients with various types of solid cancer based only on these 2 parameters has been proposed. ${ }^{45}$ However, the group $^{45}$ states that other D-dimer assays, which were not used in their studies need to be validated for use in the proposed clinical model before it can be used to predict cancer-associated VTE. ${ }^{45}$

This model has been tested by Riondino $\mathrm{S}$ et $\mathrm{al}^{46}$ by applying it to the dataset of cancer patients, who were enrolled in the Institutional Biobanks in their research. The model resulted in a similar analytical and statistic performance to those reported by Pabinger ${ }^{45}$ even with a different D-dimer assay. This indicates a large clinical applicability of the proposed model. ${ }^{46}$

Clinical suspicion with an elevated D-dimer level in cancer patients can assist in the diagnosis of a VTE as in the case of $\mathrm{PE} ;{ }^{47}$ a study showed that patients with unsuspected PE had elevated D-dimers similar to those found among cancer patients with suspected PE. ${ }^{47}$ However, other studies gave different accounts. By researching the predictive value of D-dimer of thrombosis in 67 patients with cervical cancer, it was shown that a statistically significant relationship between the levels of D-dimer and the stage of cervical cancer existed, but no significant association with PTP. Specificity and positive predictive value of the D-dimer assay used in this study is reduced in cancer patients. $^{48}$

It is possible that the incidence of VTE is overestimated, ${ }^{49}$ because the current $\mathrm{D}$-dimer reference ranges is at $(0$ $\pm 0.5 \mathrm{mg} / \mathrm{L}, 500 \mathrm{ng} / \mathrm{mL}$ ) and almost all cancer patients already exceed these limits. Therefore, these references are unsuitable for cancer. ${ }^{49}$ A cutoff value of D-dimer level at $981 \mathrm{ng} / \mathrm{mL}$ had effectively improved the exclusion of DVT by increasing the specificity from $61.82 \%$ to $85.45 \%$ without the loss of sensitivity in patients aged 40 years or younger based on a research, which included 711 cancer patients. ${ }^{50}$

In contrast, another study ${ }^{44}$ found by using ELIZA testing of D-dimer at a cutoff value $0.5 \mathrm{ug} / \mathrm{mL}$, that the difference in mean D-dimer levels between patients with VTE and patients without VTE was lowest in patients with lymphoma. A negative D-dimer test cannot safely exclude VTE in lymphoma patients, and suggested lowering the thresholds to detect VTE in both lymphoma and leukemia. ${ }^{44}$ This study ${ }^{44}$ was carried out in two independent cohorts of cancer patients, who visited the A\&E of Anderson Cancer Center, USA in the period (01.01.2009-31.12.2012), and the A\&E of King Hussein Cancer Center, Jordan in the period (01.01.2009-31.12.2015). ${ }^{44}$

A study ${ }^{51}$ involving 1178 cancer patients who were included in CAT study, showed that the high level of D-dimer is not necessarily mediated by an increased risk of VTE, but rather an indication of a more aggressive tumor biology and was associated with a poor overall survival. ${ }^{51}$ The mortality risk in patients with an elevated D-dimer remained independently increased in different subgroups after adjustment for age, sex, and VTE. ${ }^{51}$ An elevated D-dimer can be biochemically significant in predicting metastasis and progression in breast, gastric, pancreatic, colon and rectal cancer patients. ${ }^{52}$

Unprovoked VTE is related to a higher incidence of occult cancer. ${ }^{53}$ Approximately $20 \%$ of all new cases of VTE are associated with the presence of an underlying cancer. $^{54}$ A study ${ }^{54}$ which enrolled 422 first lifetime VTE; 239 DVTs and 183 PEs with a 4.7 years followup, found that there was a higher rate of cancer-incidence at D-dimer $>5000 \mathrm{ng} / \mathrm{mL}$ and the risk of cancer was 3.3-fold at D-dimer $>5000 \mathrm{ng} / \mathrm{mL}$ than those who had D-dimer $<2000 \mathrm{ng} / \mathrm{mL}$. At the time of cancer diagnosis, $80 \%$ of subjects with D-dimer $>5000 \mathrm{ng} / \mathrm{mL}$ and $20 \%$ D-dimer $<2000 \mathrm{ng} / \mathrm{mL}$ had some degree of metastasis. Lung $(29 \%)$, prostate $(21 \%)$, and hematological cancers 
(14\%) were the most common sites for establishing cancer diagnosis within one year. ${ }^{54}$

Another study, ${ }^{53}$ which followed up 169 patients with unprovoked VTE for 5.3 years found that 21/169 were diagnosed with cancer during initial hospitalization, with 3/169 being diagnosed after discharge with a median duration 104 days. 16 of the 24 diagnosed cancer patients had metastatic cancer. Elevated D-dimer concentrations $>4000$ $\mathrm{ng} / \mathrm{mL}$ are independently associated with the likelihood of occult cancer in unprovoked VTE and a likelihood of metastatic state at the time of cancer diagnosis. ${ }^{53}$ The home message is that elevated levels of D-dimer could be used as a surrogate biomarker to indicate the presence of an occult cancer or a future occurrence of cancer in cases of unprovoked VTE. ${ }^{54}$

\section{Imaging Techniques Used in Diagnosing DVT}

Contrast venography was the gold standard for DVT diagnosis, which assessed both distal and proximal deep veins of the lower extremity. ${ }^{26,55}$ Advances in technology has permitted that currently, compression ultrasonography (CUS) is the first-line imaging test being used in a suspected DVT and helped sometimes if available by color flow duplex. ${ }^{26,27,32}$ Therefore in other centers, Color Doppler (CD) has become the first line in investigating DVT. ${ }^{55,56} \mathrm{CD}$ assesses calf veins easier and could be used to diagnose PTS. ${ }^{57}$ Imaging also helps in prognostic evaluation by determining the extent of the upper part, the attachment to the wall and age of the thrombus. ${ }^{56}$

CUS also measures residual vein thrombosis (RVT), which like D-dimer, has been proposed to control the duration of anticoagulation in general, ${ }^{58}$ and individualize treatment of DVT and prevention of recurrence in cancer patients. $^{59}$ RSV has the superiority of being neither affected by factors acting on D-dimer, nor requires interrupting anticoagulation therapy. ${ }^{58}$ RVT could be used to identify a substantial subset of patients, who require a short term antithrombotic treatment; the DACUS study identified at least $78(30.2 \%)$ of the enrolled 258 patients with unprovoked DVT, who had an absence of RVT in CUS control after a 3-months therapy with VKA. ${ }^{58}$ The Extended DACUS Study; ${ }^{60}$ which excluded patients with cancer or known thrombophilia and enrolled 518 patients with idiopathic lower limbs DVT, also showed that treatment with VKA can be stopped for patients with no RVT measured at 3 months-therapy. This approach has a low risk of recurrence and a negligible risk for bleeding. ${ }^{60}$

In the Cancer-DACUS study, ${ }^{59} 30.3 \%$ (105 patients) of the 347 enrolled cancer patients with DVT had a complete resolution of the clot after 6 months of treatment with Low Molecular weight Heparin (LMWH) and only 3/105 developed recurrent VTE. ${ }^{59}$ Because cancer patients have a high risk of developing VTE, 14\% per year risk of VTE recurrence $^{58}$ and a major risk of hemorrhages, RVT measured at the end of 6 months of anticoagulation is prognostic for recurrent VTE and defines a group of patients at low risk of recurrence, therefore the extension of anticoagulation therapy is not necessary.

In the Limited CUS only the proximal deep veins of the lower extremity (ie popliteal veins or more proximal vessels) are assessed. If this type is repeated after 5 to 10 days following an initial negative proximal CUS then it is called a serial CUS. A Whole-leg CUS is a single examination of the distal and proximal deep veins of the lower extremity in the same setting. ${ }^{26-28,32,61}$

PTP by Wells' score, expertise, cost, feasibility were among the important factors to determine which CUS protocol was being used. ${ }^{26}$ The clinical relevance and anticoagulant treatment strategies of IDDVT is a major factor in debating US strategies and IDDVT will determine the future of whole-leg CUS (The Guidelines and Algorithms). ${ }^{26}$ The serial CUS could be dropped altogether and no need for a whole-leg CUS; ${ }^{61}$ symptomatic VTE occurred in less than $1 \%$ in the study of Mitsunaga $\mathrm{MM}$ et $\mathrm{al}^{61}$ after an initially normal single proximal CUS, and a whole-leg CUS has limitations in the diagnostic performance at the calf level and some of the positive findings might even be false positives. ${ }^{61}$ But high risk groups; previous VTE and/or an active malignancy, and recent hospitalization/major surgery and/or immobilization, should be treated differently. ${ }^{61}$

In the consensus report, ${ }^{32}$ the panel concluded that a single complete imaging study is the safest strategy for the diagnosis of DVT and that a complete duplex ultrasound (CDUS) is the preferred venous ultrasound test for the diagnosis of acute DVT. ${ }^{32}$ The panel recommends against the limited protocols for numerous reasons, ${ }^{32}$ which are also supported by Robert-Ebadis review; ${ }^{28}$ carrying a second limited CUS is difficult, time consuming, costly and because it is still only focusing on the proximal veins and neglecting the distal veins, ${ }^{32}$ it reveals a proximal DVT in only $1 \%$ to $5.7 \%(1.9 \%-12.8 \%){ }^{28,32}$ Also, unintended omission of the follow-up ultrasound study because of 
patient compliance can occur and lead to missed diagnosis. $^{32}$ The advantage of a whole CUS is that it can detect the IDDVT; studies employing this protocol reported higher detection of DVT because of DVT of the distal calf. $^{61}$

This review is in favor of performing a whole-leg CUS and in favor of establishing the diagnosis of IDDVT (The guidelines and Algorithms). IDDVT may play a role in the patient's ultimate outcome such as risks for recurrent DVT, APS, undiagnosed thrombophilia, subsequent diagnosis of cancer, chronic venous insufficiency etc. ${ }^{32}$ The adoption of a complete CUS examination will simplify the workup and follow-up of most patients. ${ }^{28,32}$ Also, it allows detailed assessment for alternate diagnoses, many of which (such as a muscular hematoma) can be worsened by anticoagulation. ${ }^{29}$ The panel's recommendations in the consensus report ${ }^{32}$ are more compatible with the ASH guidelines rather than NICE guidelines.

\section{Conclusion}

VTE is a complex disease, the third leading vascular disease and has a high economic impact. Finding dependable algorithms for management is a must, but they are currently non-ideal and multiple. Also, they have limitations in patients who are pregnant, elderly, on anticoagulants, having cancer and/or recurrent VTE. Unifying the used Wells' score between countries and applying 3 risk stratification Wells' criteria for DVT may decrease the incidence of missed DVT diagnosis. Also, modifying the current Wells' score by changing the risk factor from "Previously documented DVT" to "Previously documented DVT and/or PE" may increase its performance. This cautious suggestion warrants further studies.

A highly sensitive D-dimer maybe used to exclude DVT in low or moderate PTP groups. But there are many preclinical variables, which require consideration in interpreting the D-dimer test including factors that may lead to a negative result; anticoagulation therapy, small thrombi, deficiency in the fibrinolysis, etc. This case revealed the clinically relevant limitations of D-dimer testing. Therefore, referring patients with moderate PTP to imaging regardless of the result of a high quality D-dimer testing could be justifiable.

Although, D-dimer testing for detecting VTE in cancer patients has limitations at the current cutoff value $0 \pm 0.5 \mathrm{mg}$ / $\mathrm{L}$, and if the level is elevated, it could simply reflect the malignancy itself rather than VTE, very high levels of D-dimer in unprovoked VTE justifies excluding cancer at the time of VTE presentation or at least a 1 year follow-up. This cautious suggestion necessitates additional studies.
Diagnosing IDDVT is warranted because it is a frequent medical condition and it may lead to the diagnosing of APS, thrombophilia, cancer or recurrent DVT. Therefore, when imaging is indicated to exclude DVT or to identify an alternative diagnosis, referring the patient to a single complete CUS study of the lower limb is the safest strategy. Also, it is in accordance with the recommendations from the Society of Radiologists in $2016 .^{32}$

\section{Acknowledgment}

The authors sincerely thank the patient for agreeing to publish anonymously this case in an attempt to contribute to the review of the current guidelines, and the two anonymous reviewers for their careful reading of the manuscript and insightful suggestions. The authors also thank the department of Cardiology at Nordsjælland (North Zealand) University Hospital for their financial support in completing the publication of the manuscript.

\section{Funding}

This is a noncommercial research. The authors did not receive any form of funding for the presented review.

\section{Ethics}

The patient's oral consent for publication of the case details was witnessed by documenting it in the patient's official electronic system. No institutional approval was required to publish the case details.

\section{Disclosure}

The authors report no conflicts of interest in this work.

\section{References}

1. Huisman M, Klok F. Current challenges in diagnostic imaging of venous thromboembolism. Blood. 2015;126(21):2376-2382. doi:10.1182/ blood-2015-05-640979

2. Cushman M, Creager M. Improving awareness and outcomes related to venous thromboembolism. JAMA. 2015;314(18):1913. doi:10.1001/ jama.2015.15107

3. Grosse S, Nelson R, Nyarko K, Richardson L, Raskob G. The economic burden of incident venous thromboembolism in the United States: a review of estimated attributable healthcare costs. Thromb Res. 2016;137:3-10. doi:10.1016/j.thromres.2015.11.033

4. Riley R, Gilbert A, Dalton J, Pai S, McPherson R. Widely used types and clinical applications of d-dimer assay. Lab Med. 2016;47 (2):90-102. doi:10.1093/labmed/lmw001

5. American Society of Hematology. ASH releases new clinical practice guidelines for venous thromboembolism: evidence-based guidelines to drive improved care for patients with blood clots. hematology.org. Available from: https://www.hematology.org/newsroom/press-releases /2018/ash-releases-new-clinical-practice-guidelines-venous-thromboem bolism. Accessed July 20, 2019. 
6. Heit JA. Epidemiology of venous thromboembolism. Nat Rev Cardiol. 2015;12(8):464-474. doi:10.1038/nrcardio.2015.83

7. Fernandez MM, Hogue S, Preblick R, Kwong WJ. Review of the cost of venous thromboembolism. Clinicoecon Outcomes Res. 2015;7:451-462. doi: $10.2147 /$ ceor.s85635

8. Chappell F, Andras A, Welch K, et al. D-Dimer tests for the diagnosis of deep venous thrombosis in symptomatic hospital outpatients with a clinical prediction rule. Cochrane Database Syst Rev. 2016;9. doi:10.1002/14651858.cd012356

9. Prochaska J, Frank B, Nagler M, et al. Age-related diagnostic value of D-dimer testing and the role of inflammation in patients with suspected deep vein thrombosis. Sci Rep. 2017;7(1):4591. doi:10.1038/s41598-017-04843-x

10. Patel K Deep venous thrombosis (DVT): practice essentials, background, anatomy. emedicine.medscape.com. Available from: https://emedicine. medscape.com/article/1911303-overview. Accessed July 27, 2019.

11. Le Gal G, Righini M. Controversies in the diagnosis of venous thromboembolism. J Thromb Haemost. 2015;13(Suppl 1):S259S265. doi:10.1111/jth.12937

12. Lo B, Brenner B deep venous thrombosis risk stratification: risk stratification. emedicine.medscape.com. Available from: https://emedicine. medscape.com/article/1918446-overview. Accessed July 27, 2019.

13. Lim W, Le Gal G, Bates S, et al. American society of hematology 2018 guidelines for management of venous thromboembolism: diagnosis of venous thromboembolism. Blood Adv. 2018;2(22):3226-3256. doi:10.1182/bloodadvances. 2018024828

14. Ekelund S Why D-dimer tests cannot be used to exclude venous thromboembolism in patients with high pretest probability. Acutecaretesting.org. Available from: https://acutecaretesting.org/en/ articles/why-ddimer-tests-cannot-be-used-to-exclude-venousthromboembolism-in-patients. Accessed July 28, 2019.

15. Lægehåndbogen. Dyb vene trombose, DVT - Klinisk scoringssystem (Wells score). Sundhed.dk. Available from: https://www.sundhed.dk/sund hedsfaglig/laegehaandbogen/hjerte-kar/tilstande-og-sygdomme/trom boembolisk-sygdom/dyb-venetrombose-dvt/. Accessed August 5, 2019.

16. Wells P Wells' criteria for DVT - MDCalc. mdcalc.com. Available from: https://www.mdcalc.com/wells-criteria-dvt. Accessed August $5,2019$.

17. Gurram M, Pulivarthi S. Effectiveness of D-dimer as a screening test for venous thromboembolism: an update. $N$ Am J Med Sci. 2014;6 (10):491-499. doi:10.4103/1947-2714.143278

18. Bounds E, Kok S, Dimer D. In: statPearls [internet]. Treasure island (FL): statPearls. Available from: Ncbi.nlm.nih.gov.https://www.ncbi. nlm.nih.gov/books/NBK431064/. Accessed July 30, 2019.

19. Oxford University Hospitals, NHS Foundation Trust. Oxford haemophilia and thrombosis center out-patient DVT service protocol. oxford: oxford university hospitals; 2019. version 9.6 [updated by curry N]. Available from: https://www.ouh.nhs.uk/services/referrals/ specialist-medicine/documents/dvt-protocols.pdf. Accessed April 30, 2020

20. Roy P, Meyer G, Vielle B, et al. Appropriateness of diagnostic management and outcomes of suspected pulmonary embolism. Ann Intern Med 2006;144(3):157-164. doi:10.7326/0003-4819-144-3-200602070-00003

21. Giannitsis E, Mair J, Christersson C, et al. How to use D-dimer in acute cardiovascular care. Eur Heart J. 2016;6(1):69-80. doi: $10.1177 / 2048872615610870$

22. Sevestre M, Quashié C, Genty C, Rolland C, Quéré I, Bosson J. Clinical presentation and mortality in pulmonary embolism: the Optimev study. J Mal Vasc. 2010;35(4):242-249. doi:10.1016/j.jmv.2010.05.004

23. Gordon N, Schreiber M. Pulmonary emboli and deep vein thromboses: are they always part of the same disease spectrum? Mil Med. 2016;181(5S):104-110. doi:10.7205/milmed-d-15-00156

24. Palareti G, Antonucci E, Dentali F, et al. Patients with isolated pulmonary embolism in comparison to those with deep venous thrombosis. Differences in characteristics and clinical evolution. Eur J Intern Med. 2019;69:64-70. doi:10.1016/j.ejim.2019.08.023
25. Sartori M, Cosmi B, Legnani C, et al. The Wells rule and D-dimer for the diagnosis of isolated distal deep vein thrombosis. $J$ Thromb Haemost. 2012;10(11):2264-2269. doi:10.1111/j.1538-7836.2012.04895.x

26. Kraaijpoel N, Carrier M, Le Gal G, et al. Diagnostic accuracy of three ultrasonography strategies for deep vein thrombosis of the lower extremity: a systematic review and meta-analysis. PLoS One. 2020;15(2):e0228788. doi:10.1371/journal.pone.0228788

27. Palareti G. How I treat isolated distal deep vein thrombosis (IDDVT). Blood. 2014;123(12):1802-1809. doi:10.1182/blood-2013-10-512616

28. Robert-Ebadi H, Righini M. Should we diagnose and treat distal deep vein thrombosis? Hematology. 2017;2017(1):231-236. doi:10.1182/ asheducation-2017.1.231

29. Horner D, Hogg K, Body R. Should we be looking for and treating isolated calf vein thrombosis? Emergency Med J. 2015;33 (6):431-437. doi:10.1136/emermed-2014-204230

30. Palareti G, Agnelli G, Imberti D, et al. A commentary: to screen for calf DVT or not to screen? The highly variable practice among Italian centers highlights this important and still unresolved clinical option. results from the Italian MASTER registry. Thromb Haemost. 2008;99 (01):241-244. doi:10.1160/th07-06-0393

31. Asbeutah A, Riha A, Cameron J, McGrath B. Five-year outcome study of deep vein thrombosis in the lower limbs. J Vasc Surg. 2004;40(6):1184-1189. doi:10.1016/j.jvs.2004.10.025

32. Needleman L, Cronan J, Lilly M, et al. Ultrasound for lower extremity deep venous thrombosis. Multidisciplinary recommendations from the society of radiologists in ultrasound consensus conference. Circulation. 2018;137(14):1505-1515. doi:10.1161/circulationaha.117.030687

33. Der Sahakian G, Claessens Y, Allo J, Kansao J, Kierzek G, Pourriat J. Accuracy of D-dimers to rule out venous thromboembolism events across age categories. Emerg Med Int. 2010;2010:1-4. doi:10.1155/ 2010/185453

34. Wells P, Anderson D, Rodger M, et al. Evaluation of D-dimer in the diagnosis of suspected deep-vein thrombosis. New Engl J Med. 2003;349(13):1227-1235. doi:10.1056/nejmoa023153

35. Dagkiran H, Atas R, Tomic I, et al. Severe pulmonary embolism with negative D-DIMER-TESTING. $J$ Cardiol Therapy. 2015;2(1): Available from. http://www.ghrnet.org/index.php/jct/article/view/ 1033/1164. Accessed: August 5, 2019.

36. Singer A, Zheng H, Francis S, et al. D-dimer levels in VTE patients with distal and proximal clots. Am J Emerg Med. 2019;37(1):33-37. doi:10.1016/j.ajem.2018.04.040

37. Baglin T, Keeling D, Kitchen S. Effects on routine coagulation screens and assessment of anticoagulant intensity in patients taking oral dabigatran or rivaroxaban: guidance from the British committee for standards in haematology. Br J Haematol. 2012;159(4):427-429. doi:10.1111/bjh. 12052

38. Couturaud F, Kearon C, Bates S, Ginsberg J. Decrease in sensitivity of D-dimer for acute venous thromboembolism after starting anticoagulant therapy. Blood Coagulation Fibrinolysis. 2002;13 (3):241-246. doi:10.1097/00001721-200204000-00010

39. Niimi R, Hasegawa M, Shi D, Sudo A. The influence of fondaparinux on the diagnosis of postoperative deep vein thrombosis by soluble fibrin and D-dimer. Thromb Res. 2012;130(5):759-764. doi:10.1016/ j.thromres.2011.11.046

40. Legnani C, Martinelli I, Palareti G, et al. D-dimer levels during and after anticoagulation withdrawal in patients with venous thromboembolism treated with non-vitamin $\mathrm{K}$ anticoagulants. PLoS One. 2019;14(7):e0219751. doi:10.1371/journal.pone.0219751

41. Kawai T, Kuroda Y, Goto K, Matsuda S. Does prophylactic administration of edoxaban increase d-dimer levels after total hip arthroplasty? J Clin Med. 2019;8(5):678. doi:10.3390/ jcm8050678

42. Wu C, Chen B, Wang J, Yen S, Huang C. Plasma D-dimer is not useful in the prediction of deep vein thrombosis after total knee arthroplasty in patients using rivaroxaban for thromboprophylaxis. J Orthop Surg Res. 2018;13(1). doi:10.1186/s13018-018-0883-1 
43. Allaham H, Mansour A, Abdullah O, Danila C. D-dimer sensitivity in fully anticoagulated patients with moderate pretest probability for pulmonary thromboembolism. J Am Coll Cardiol. 2019;73(9):2168. doi:10.1016/s0735-1097(19)32774-3

44. Qdaisat A, Soud R, Wu C, et al. Poor performance of D-dimer in excluding venous thromboembolism among patients with lymphoma and leukemia. Haematologica. 2019;104(6):e265-e268. doi:10.3324/ haematol.2018.211466

45. Pabinger I, van Es N, Heinze G, et al. A clinical prediction model for cancer-associated venous thromboembolism: a development and validation study in two independent prospective cohorts. Lancet Haematol. 2018;5(7):e289-e298. doi:10.1016/s2352-3026(18)30063-2

46. Riondino S, Ferroni P, Zanzotto F, Roselli M, Guadagni F. Predicting VTE in cancer patients: candidate biomarkers and risk assessment models. Cancers. 2019;11(1):95. doi:10.3390/cancers11010095

47. Ali S, Dilday E, Tagawa S, et al. D-dimer levels among cancer patients with unsuspected pulmonary embolism: clinical correlates and relevance. Blood. 2012;120(21):1154. doi:10.1182/blood.v120.21.1154.1154

48. Zayyan M, Katung G, Abdul-Aziz H, Igashi J D-dimer levels as marker of deep vein thrombosis in patients with cervical cancer: a study of a northern Nigerian patient population. Smjonline.org. Available from: http://www.smjonline.org/text.asp?2019/22/2/86/ 260840. Accessed March 1, 2020.

49. Yu J, Li D, Lei D, et al. Tumor-specific D-dimer concentration ranges and influencing factors: a cross-sectional study. PLoS One. 2016;11 (11):e0165390. doi:10.1371/journal.pone.0165390

50. Chen C, Li G, Liu Y, Gu Y. A new D-dimer cutoff value to improve the exclusion of deep vein thrombosis in. Cancer Patients. 2020. doi:10.7314/APJCP.2014.15.4.1655

51. Ay C, Dunkler D, Pirker R, et al. High D-dimer levels are associated with poor prognosis in cancer patients. Haematologica. 2012;97 (8):1158-1164. doi:10.3324/haematol.2011.054718

52. Dai H, Zhou H, Sun Y, et al. D-dimer as a potential clinical marker for predicting metastasis and progression in cancer. Biomed Rep. 2018;9(5):453-457. doi:10.3892/br.2018.1151
53. Han D, Hartaigh B, Lee J, et al. Impact of D-dimer for prediction of incident occult cancer in patients with unprovoked venous thromboembolism. PLoS One. 2016;11(4):e0153514. doi:10.1371/ journal.pone.0153514

54. Gran O, Braekkan S, Paulsen B, Skille H, Hansen J. D-dimer measured at first venous thromboembolism is associated with future risk of cancer. Haematologica. 2016;101(12):e473-e475. doi:10.3324/ haematol.2016.151712

55. Awad I. Value of D ultrasound in diagnosis of clinically suspected deep vein thrombosis. IOSR J Dent Med Sci. 2018.

56. Mohammed S, AL-Najjar S. Role of color flow ultrasound in detection of deep venous thrombosis. J Educ Pract. 2016;27(4):2222.

57. Michiels J, Moosdorff W, Lao M, et al. Diagnosis and treatment of DVT and prevention of DVT recurrence and the PTS: bridging the gap between DVT and PTS in the primary care setting or outpatient ward. J Vasc Diagn Interv. 2017;5:21-34. doi:10.2147/jvd. s62734

58. Malato A, Saccullo G, Iorio A, Ageno W, Siragusa S. Residual vein thrombosis and d-dimer for optimizing duration of anticoagulation in idiopathic deep vein thrombosis. Curr Pharm Des. 2010;16 (31):3483-3486. doi:10.2174/138161210793563428

59. Napolitano M, Saccullo G, Malato A, et al. Optimal duration of low molecular weight heparin for the treatment of cancer-related deep vein thrombosis: the cancer-DACUS study. J Clin Oncol. 2014;32 (32):3607-3612. doi:10.1200/JCO.2013.51.7433

60. Siragusa S, Malato A, Bellisi M, et al. Absence of residual vein thrombosis after an episode of idiopathic deep vein thrombosis: short-term anticoagulation is safe. the "extended dacus study". Blood. 2007;110(11):301. doi:10.1182/blood.v110.11.301.301

61. Mitsunaga M, Kogachi S, Yoon H. Risk of venous thromboembolism after a single normal proximal lower extremity venous ultrasound. Perm J. 2017;21:16-140. doi:10.7812/tpp/16-140
Journal of Blood Medicine

\section{Publish your work in this journal}

The Journal of Blood Medicine is an international, peer-reviewed, open access, online journal publishing laboratory, experimental and clinical aspects of all aspect pertaining to blood based medicine including but not limited to: Transfusion Medicine; Blood collection, Donor issues, Transmittable diseases, and Blood banking logistics; Immunohematology; Artificial and alternative blood based therapeutics; Hematology; Biotechnology/nanotechnology of blood related medicine; Legal aspects of blood medicine; Historical perspectives. The manuscript management system is completely online and includes a very quick and fair peer-review system. Visit http://www.dovepress.com/testimonials.php to read real quotes from published authors. 